\title{
A Non-invasive Focused Extracorporeal Shock Wave Therapy System Promotes Increased Tissue Oxygen Saturation in Chronic Wounds in Persons with Diabetes
}

\author{
Windy Cole ${ }^{1, \text { * }}$, Stacey Coe $^{1}$, Greg Maislin ${ }^{2}$, Valerie Marmolejo ${ }^{3}$ \\ ${ }^{1}$ College of Podiatric Medicine, Kent State University, Independence, the United States \\ ${ }^{2}$ Biomedical Statistical Consulting, Wynnewood, the United States \\ ${ }^{3}$ Scriptum Medica, University Place, Washington, the United States
}

Email address:

WCole4@kent.edu (W. Cole), Scoe3@kent.edu (S. Cole), Gregmaislin@biomedstat.com (G. Maislin), Vlsdpm@gmail.com (V. Marmolejo)

${ }^{*}$ Corresponding author

\section{To cite this article:}

Windy Cole, Stacey Coe, Greg Maislin, Valerie Marmolejo. A Non-invasive Focused Extracorporeal Shock Wave Therapy System Promotes Increased Tissue Oxygen Saturation in Chronic Wounds in Persons with Diabetes. American Journal of Nursing Science.

Vol. 10, No. 3, 2021, pp. 156-162. doi: 10.11648/j.ajns.20211003.14

Received: April 27, 2021; Accepted: May 8, 2021; Published: May 20, 2021

\begin{abstract}
Diabetes affects 30.3 million Americans and is on the rise. Chronic lower extremity ulceration in patients with diabetes is a common complication that can lead to major morbidity and mortality if not addressed expeditiously. Despite numerous advances in wound care and healing over the past two decades, these ulcerations continue to pose a significant clinical problem. This case series aimed to evaluate the effectiveness of focused extracorporeal shock wave therapy (F-ESWT) administered via a pulsed acoustic cellular expression system in enhancing localized wound tissue oxygenation and perfusion in chronic wounds. Fifteen subjects with a history of diabetes and a chronic wound of the foot or ankle that had failed to achieve wound area reduction of $50 \%$ or greater after at least 4 weeks of standard of care treatment underwent four weekly treatments of F-ESWT administered via a pulsed acoustic cellular expression system. Near-infrared spectroscopy (NIRS) was used to determine the effectiveness of F-ESWT on tissue oxygen saturation within the wound bed. All subjects demonstrated a statistically significant increase in tissue oxygen saturation within the wound bed as well as a decrease in wound area. Seven wounds healed. Results of this case study suggest that F-ESWT promotes increased tissue oxygenation within the wound bed and neovascularization, potentially facilitating accelerated wound resolution of chronic lower extremity ulcerations in patients with diabetes.
\end{abstract}

Keywords: Extracorporeal Shock Wave Therapy, Diabetic feet, Diabetic Foot Ulcer, Angiogenesis, Spectroscopy, Near-Infrared, Oxygenation, Oxygenated Hemoglobin, Neovascularization

\section{Introduction}

The diabetes epidemic continues to grow in the United States (US). The most recent National Diabetes Statistics Report released by the Centers for Disease Control estimates that 30.3 million Americans, or roughly one out of every ten US citizens, are living with diabetes, and this number is rising [1]. Possible long-term complications of uncontrolled diabetes include damage to large (macrovascular) and small (microvascular) blood vessels. Such damage can lead to, among other morbidities, neuropathy and the development of non-healing wounds, particularly on the lower extremities.
Despite advancements in medicine over the past two decades, chronic lower extremity ulcerations in those with diabetes continue to pose a significant clinical problem [2]. It is estimated that $85 \%$ of all non-traumatic lower extremity amputations are a direct result of these types of ulcerations [3].

Ischemia, microcirculatory dysfunction, and peripheral vascular disease (PVD) cause limitations in blood flow that can delay the healing process and result in a high-risk for lower extremity amputation in patients with diabetes [4]. Advancements in the understanding of the physiology of neovascularization have led researchers to examine the effects of external physical force manipulation on tissues to increase 
new vessel growth and improve wound healing. One such modality is Focused Extracorporeal Shock Wave Therapy (F-ESWT) [5].

F-ESWT is a non-invasive treatment that can be safely and easily performed in the outpatient setting as an adjunctive therapy. F-ESWT uses the electrohydraulic shockwave principle to deliver focused, biphasic, high-energy acoustic pressure waves [6]. Research has shown that the local delivery of focused shock wave therapy stimulates the early expression of angiogenesis-related growth factors, including endothelial nitric oxide synthase (eNOS), vascular endothelial growth factor (VEGF) and proliferating cell nuclear antigen (PCNA) [7]. These effects of F-ESWT have been demonstrated to have healing effects in both bone and soft tissue [8].

Several methods can used to measure perfusion and wound tissue oxygenation. One of these is a novel near-infrared spectroscopy (NIRS) imaging device that objectively measures the tissue oxygenation saturation percentage $\left(\mathrm{S}_{\mathrm{t}} \mathrm{O}_{2} \%\right)$ of the wound bed. Hyperspectral technology has been successfully used in previous clinical studies to evaluate functional tissue oxygen saturation in the management of diabetic foot ulcerations [9-11].

In this case series the authors hypothesized that the administration of F-ESWT via a pulsed acoustic cellular expression system (dermaPACE ${ }^{\circledR}$ System, SANUWAVE, Suwanee, GA) over four (4) consecutive weeks would result in a significant increase in the oxygenated hemoglobin ratio in the wound bed which would potentially increase wound healing. A NIRS device (Snapshot ${ }_{\mathrm{NIR}}$ Kent Imaging, Calgary, $\mathrm{AB}$, Canada) was used to track tissue oxygen saturation.

\section{Methods}

This case series reports on fifteen wounds observed in the primary author's clinical practice between January 2019 and December 2019. Patients were all $\geq 18$ years of age with an ankle-brachial index between $\geq 0.9 \mathrm{mmHg}$ and $<1.4 \mathrm{mmHg}$, and an $\mathrm{HbA1c}$ level between 7-12\%. All fifteen wounds had a minimum duration of 4 weeks, had failed to achieve at least $50 \%$ wound area reduction after a minimum of 4 weeks treatment with standard of care wound therapy, and were negative for clinical signs and symptoms of infection. There was no upper or lower limit to wound size. Previous wound management varied and included debridement, offloading, compression bandages, alginates, collagen, gelling hydrofibers, and foam dressings. All patients provided written informed consent to publish the case details and associated de-identified image assessments.

Patients were seen weekly for five consecutive visits and received a total of four F-ESWT treatments unless they healed earlier. An FDA cleared, non-invasive pulsed acoustic cellular expression system (dermaPACE ${ }^{\circledR}$ System, SANUWAVE, Suwanee, GA) was used to provide F-ESWT treatment. Therapy was administered per the manufacturer's guidelines in which wound size determines the number of shocks administered per treatment (Figure 1).

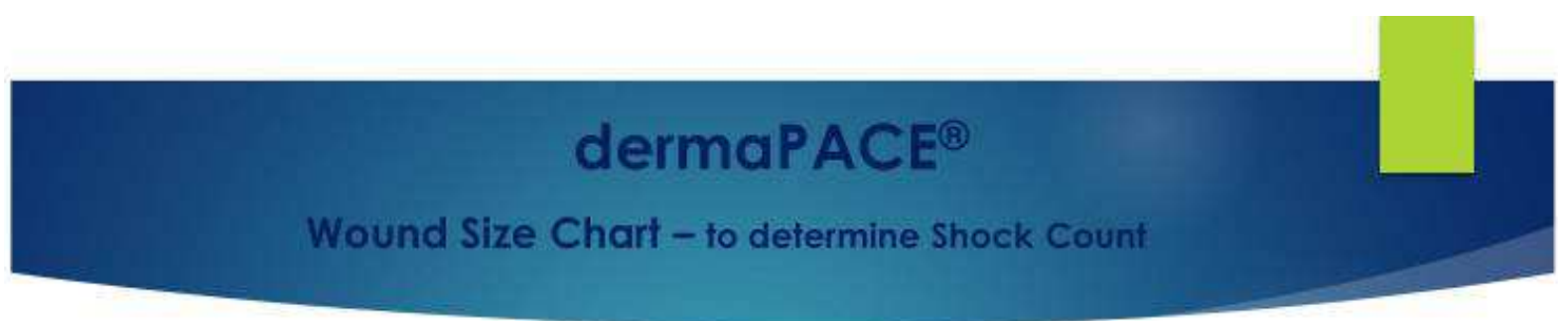

PACE treatment was administered per the manufacturer's guidelines in which wound size determines the number of shocks administered per treatment

\begin{tabular}{|l|c|c|c|c|c|c|c|c|c|c|}
\begin{tabular}{|l} 
Wound Area \\
$\left(\mathrm{cm}^{2}\right)$
\end{tabular} & $\leq 1.9$ & $\begin{array}{c}2.0- \\
2.9\end{array}$ & $3.0-3.9$ & $\begin{array}{c}5.0- \\
4.9\end{array}$ & $5.0-$ & $5.9-6.9$ & $7.0-7.9$ & $8.0-8.9$ & $\begin{array}{c}9.0- \\
9.9\end{array}$ & $\begin{array}{c}10.0- \\
10.9\end{array}$ \\
\hline $\begin{array}{c}\text { Number of } \\
\text { Shocks }\end{array}$ & 1000 & 1100 & 1200 & 1300 & 1400 & 1500 & 1600 & 1700 & 1800 & 1900 \\
\hline $\begin{array}{l}\text { Wound } \\
\text { Area }\left(\mathrm{cm}^{2}\right)\end{array}$ & $11.0-11.9$ & $12.0-12.9$ & $13.0-13.9$ & $14.0-14.9$ & $15.0-15.9$ & $16.0-16.9$ \\
\hline $\begin{array}{c}\text { Number of } \\
\text { Shocks }\end{array}$ & 2000 & 2100 & 2200 & 2300 & 2400 & 2500 \\
\hline
\end{tabular}

Figure 1. Determination of shock count per treatment with the dermaPACE® system based on wound size.

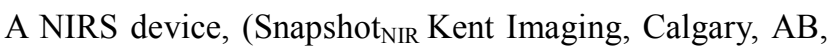
Canada) was used to assess localized wound tissue oxygenation and perfusion. NIRS provided immediate photographic image capture and point-of-care site specific information to objectively track changes in $\mathrm{S}_{\mathrm{t}} \mathrm{O}_{2} \%$. At baseline, prior to the first F-ESWT treatment, wounds were measured manually, a NIRS image of the wound was taken, and the NIRS system was directed to calculate the $\mathrm{S}_{\mathrm{t}} \mathrm{O}_{2} \%$ at a standardized central point within the wound bed. The same procedures were repeated prior to each subsequent weekly 
F-ESWT treatment. Final wound measurements, NIRS images and $\mathrm{S}_{\mathrm{t}} \mathrm{O}_{2} \%$ were obtained at Week 5 after completion of four F-ESWT treatments, or upon wound resolution if complete healing occurred prior to Week 5 .

During the treatment period, all wounds were treated with the standard of care. Debridement was done when clinically indicated. Bandages consisted of inert dressing materials (plain foam or alginate and dry secondary dressings) to manage exudate and promote a moist wound healing environment. Offloading via total contact casting (TCC) was used in the treatment of any plantar foot wounds. During the treatment period, no patient underwent any other intervention to improve circulation. Healing was defined as $100 \%$ re-epithelization with no drainage and no need for dressing application.

\section{Statistical methods}

The wound area tissue oxygen saturation percentage were quantitatively described at baseline and follow-up using descriptive statistics (mean, SD, median, minimum and maximum). Descriptive statistics (mean, SD, median, minimum and maximum) and $95 \%$ confidence intervals (CI) were used to describe absolute and percent change in the oxygenated hemoglobin ratio from baseline to study completion and in wound area from baseline to study completion. A two-sided paired t-test ( $\alpha=0.05$ ) was used to test the research hypothesis that the mean change in tissue oxygen saturation and in wound area was larger than zero. A Spearman Rank Correlation test $(\alpha=0.05)$ was used to test for an association between change in $\mathrm{S}_{\mathrm{t}} \mathrm{O}_{2} \%$ and wound area. All statistical analyses were performed using SAS Version 9.4 (SAS Institute Inc., Cary, NC, USA).

\section{Results}

The patient cohort consisted of fourteen subjects, eight male and six female, with a chronic foot or ankle wound and a diagnosed history of diabetes. One male patient had two wounds - one on the medial aspect of his foot, and one on the lateral. Mean patient age was $64.5 \pm 10.2$ years (range: 49 to 88). Mean wound area at baseline was $21.65 \pm 30.85 \mathrm{~cm}^{2}$ (range: 0.42 to 93.10 ). Due to the large variation in wound size, average values were also evaluated in terms of medians. The median baseline wound area was $7.5 \mathrm{~cm}^{2}$. Two of $15(13.3 \%)$ wounds had a baseline wound area $<1 \mathrm{~cm}^{2}$. Nine of $15(60 \%)$ of the wounds had a wound area $>7 \mathrm{~cm}^{2}$. The average wound duration prior to beginning weekly F-ESWT treatments was $12.33 \pm 5.3$ weeks. Mean baseline $\mathrm{S}_{t} \mathrm{O}_{2}$ was $60.7 \pm 18.9 \%$ (Table 1).

Table 1. Patient demographics and Baseline and Final $\mathrm{S}_{t} \mathrm{O}_{2} \%$ and Wound Area.

\begin{tabular}{|c|c|c|c|c|c|c|}
\hline Gender & Age & Wound Duration at Study Entry (weeks) & Baseline $\mathrm{S}_{\mathrm{t}} \mathrm{O}_{2} \%$ & Final $\mathrm{S}_{\mathrm{t}} \mathrm{O}_{2} \%$ & Baseline Area $\left(\mathrm{cm}^{2}\right)$ & Final Wound Area $\left(\mathrm{cm}^{2}\right)$ \\
\hline $\mathrm{F}$ & 49 & 6 & 58 & 71 & 1.6 & Healed \\
\hline $\mathrm{F}$ & 56 & 16 & 49 & 60 & 93.1 & 82.65 \\
\hline M & 56 & 14 & 69 & 77 & 1.7 & Healed \\
\hline $\mathrm{F}$ & 57 & 6 & 74 & 86 & 9.8 & 5.3 \\
\hline M & 58 & 11 & 47 & 77 & 7.0 & Healed \\
\hline $\mathrm{F}$ & 60 & 9 & 84 & 92 & 0.4 & Healed \\
\hline $\mathrm{F}$ & 61 & 21 & 57 & 72 & 70.7 & 66.24 \\
\hline $\mathrm{F}$ & 62 & 8 & 69 & 71 & 7.5 & Healed \\
\hline M & 67 & 15 & 34 & 81 & 16.4 & 0.2 \\
\hline M & 67 & 12 & 48 & 81 & 5.0 & 2.4 \\
\hline M & 70 & 9 & 82 & 85 & 0.6 & Healed \\
\hline $\mathrm{F}$ & 73 & 10 & 82 & 86 & 3.0 & 0.1 \\
\hline $\mathrm{F}$ & 81 & 7 & 20 & 52 & 9.5 & 7.1 \\
\hline M & 88 & 22 & 80 & 83 & 24.4 & Healed \\
\hline
\end{tabular}

Upon study completion, all 15 wounds demonstrated a decrease in wound area, with seven (46.7\%) having healed. The mean final wound area, including healed wounds, was $15.9 \pm 29.18 \mathrm{~cm}^{2}$. The mean final wound area, excluding healed wounds, was $28.49 \pm 35.65 \mathrm{~cm}^{2}$. The mean change in wound size, including healed wounds, was $-6.46 \pm 6.65 \mathrm{~cm}^{2}$ (CI -10.14 to -2.77). When healed wounds were excluded, the mean change in wound area was $-6.71 \pm 5.02 \mathrm{~cm}^{2}$ (CI-10.91 to -2.51). Paired t-test p-values were 0.002 and 0.007 , respectively, demonstrating significant reduction in wound areas with or without excluding healed wounds. Given the large variability in wound sizes, the wound area data was also evaluated non-parametrically. The median wound area at the final study visit, including healed wounds was $0.12 \mathrm{~cm}^{2}$ (range: 0.00-82.65) and excluding resolved wounds was $6.20 \mathrm{~cm}^{2}$ (range: 0.12 to 82.65 ). The median change in wound area from baseline to final visit, including resolved wounds was -4.44 $\mathrm{cm}^{2}$ (range: -24.42 to -0.42 ) and excluding healed wounds was $-4.5 \mathrm{~cm}^{2}$ (range: -16.20 to -2.36 ).

All 15 wounds displayed a significant increase in wound bed tissue oxygen saturation upon study completion. The average final $\mathrm{S}_{\mathrm{t}} \mathrm{O}_{2} \%$ was $75.7 \pm 11.1 \%$ with an absolute change of $15.07 \pm 13.8 \%$. Statistical significance was seen with a two-sided paired t-test $(\mathrm{df}=14, \mathrm{p}=0.0008, \mathrm{CI}=7.4$ to 22.7\%). A non-parametric Wilcoxon signed rank test demonstrated that this result was robust $(p<0.0001)$ and not due to a single outlier with an absolute increase of $47 \%$. The mean percentage increase of $\mathrm{S}_{\mathrm{t}} \mathrm{O}_{2} \%$ from baseline to follow up was $37.6 \pm 49.8 \% \quad(p=0.011$ - two-sided paired t-test; $p$ $<0.0001$ - signed rank test; CI 10.0 to 65.1 ). In this sample, there was no association between changes in the oxygenated hemoglobin ratio and changes in wound size (all wounds, $\mathrm{N}=15$, Spearman rank correlation $=0.01, \mathrm{p}=0.96$ ).

No device-related adverse events occurred. There were no 
patient or investigator-reported complaints associated with receiving or providing F-ESWT therapy.

Case Example [6]

A 56-year-old patient with a medical history of Type 1 diabetes, neuropathy, calciphylaxis, renal dysfunction, degenerative joint disease, and hypertension, had a chronic wound of 16 weeks duration on their left ankle and foot. Baseline wound measurements were $9.5 \mathrm{~cm} \times 9.8 \mathrm{~cm}$, $\left(93.1 \mathrm{~cm}^{2)}\right.$ and the baseline percent tissue oxygen saturation at the center of the wound bed was $49 \%$ (Figure 2).

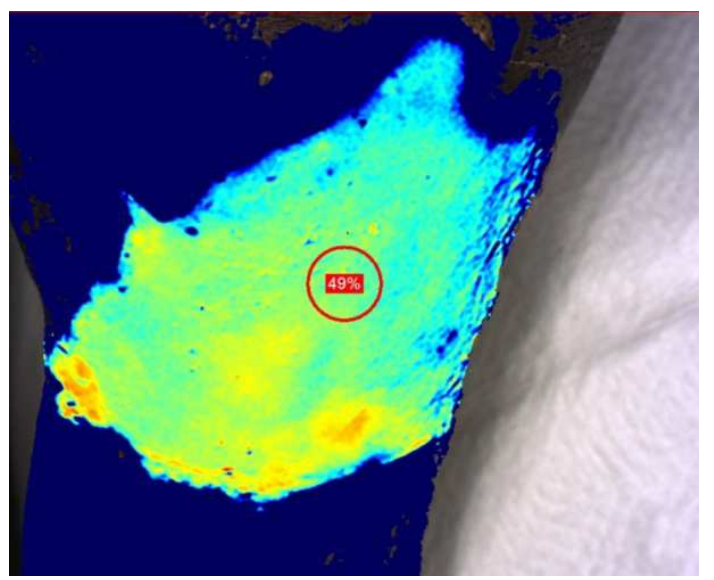

Figure 2. [6]: Baseline NIRS wound image with percent tissue oxygenation saturation calculated at the center of the wound bed.

Reproduced with permission from Cole W, Coe S., Using a non-invasive pulsed acoustic cellular expression system to promote angiogenesis in chronic wounds; published by HMP, 2020.

The patient was treated with weekly F-ESWT per the manufacturer's guidelines. After four (4) weekly F-ESWT treatments the wound reduced in size to $8.7 \mathrm{~cm} \times 9.5 \mathrm{~cm}$ $\left(82.65 \mathrm{~cm}^{2}\right)$. The percent tissue oxygen saturation at the center of the wound bed increased to $60 \%$ (Figure 3 ).

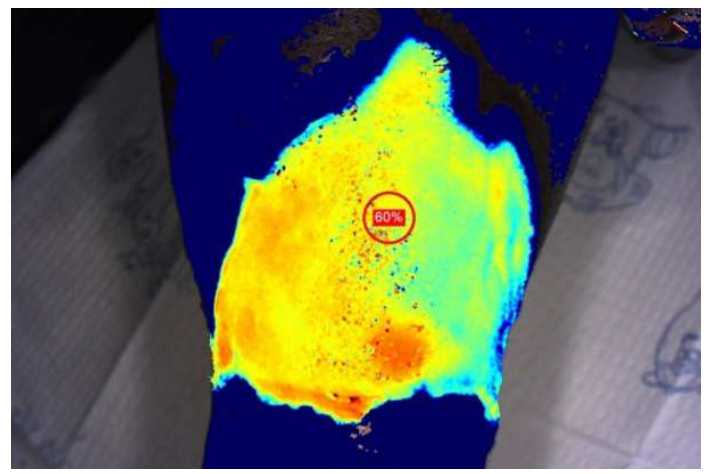

Figure 3. [6]: Final NIRS wound image with percent tissue oxygenation saturation calculated at the center of the wound bed.

Reproduced with permission from Cole W, Coe S., Using a non-invasive pulsed acoustic cellular expression system to promote angiogenesis in chronic wounds; published by HMP, 2020.

These changes translate to an $11.2 \%$ decrease in wound size, and a $22.5 \%$ improvement in $\mathrm{S}_{\mathrm{t}} \mathrm{O}_{2} \%$.

\section{Discussion}

Wound healing is an intricate multi-step process that requires essential cellular and acellular mechanisms working in tandem to restore function to the injured tissue. Wounded tissues require a significantly greater perfusion level to support healing than that required to sustain intact tissues [12]. In patients with diabetes, elevated blood glucose levels can lead to dysfunction of the microvasculature. Weakening of small blood vessel walls eventually causes decreased blood flow, diminishing the body's ability to deliver essential cellular components to wounded tissues [13]. Therapies such as F-ESWT that result in new blood vessel in-growth may improve blood supply, increase tissue oxygenation, enhance cell proliferation, and accelerate wound regeneration and healing. The results of this case series reinforce and expand on the results of prior research that suggest that F-ESWT therapy supports neovascularization resulting in increased oxygen saturation levels in wounded tissues $[5,14]$.

Non-invasive F-ESWT works by improving microcirculation and thus delivery of oxygen and other nutrients essential for wound resolution by causing the wound to transition from the inflammatory to the proliferative phase of the wound healing cascade [15]. It is known that the shockwaves that the device generates are converted from an electrical to a mechanical force that produces compressive and tensile stresses on wounded tissues resulting in a pro-angiogenic response at the cellular level [16]. Wang reported that extracorporeal shock waves (ESW), including F-ESWT cause shear forces within tissues that initiate a biological response at a cellular level producing angiogenic growth factors, including eNOS, VEGF, and PCNA [5, 14, 17]. Wang's studies also reported a significant increase in blood flow perfusion rates with ESWT treatment compared to those wounds treated with hyperbaric oxygen therapy [14, 17]. Krokowicz et al showed that F-ESWT treatment relaxes local arterioles and increases their diameter $[18,19]$. This effect, in combination with unaltered blood flow, results in better perfusion and oxygenation. Up-regulation of VEGF and von Wilenbrand Factor (vWF) expression on the endothelial cells was observed and is an indication of endothelial cell proliferation in advance of angiogenesis [18, 19]. VEGF is one of the critical components associated with angiogenesis in that it enhances proliferation and migration of endothelial cells and prevents them from apoptosis. It also participates in the up-regulation of nitric oxide (NO) synthase activity in endothelial cells. Endothelium derived NO is shown to be involved in vascular remodeling and angiogenesis [20]. In addition, Krokowicz confirmed up-regulation of key chemokines that are involved in pro-angiogenic activity in studies that demonstrated up-regulation of CCL2 pro-angiogenic gene expression and its receptor CCR2 at 24 hours after F-ESWT treatment [18, 19]. CCL2 links with angiogenic activity via recruitment of macrophages which are known to contribute to synthesis of proangiogenic factors such as VEGF $[18,19]$.

The increase in perfusion is important as it is, by definition, 
a decrease in the ischemia (lack of blood flow) that is often associated with impaired wound healing. Often wound tissues lack adequate oxygen gas exchange because blood oxygen levels are low in the wound area. In a study to determine the effect of shockwaves on microcirculatory improvement in patients with Chronic Limb Ischemia (CLI), De Sanctis et al delivered six shockwave treatments over a two-week period with an additional six weeks of follow-up visits [21]. Microcirculatory measurements were taken with Laser Doppler Imaging and $\mathrm{PO}_{2}$ and $\mathrm{PCO}_{2}$ were measured with a Kontron Analyzer [21]. De Sanctis et al reported an increase in partial pressures of oxygen gas in the blood stream $(p<0.05)$ and a decrease in carbon dioxide $(\mathrm{p}<0.05)$ during the 8 week long study [21]. Wang et al conducted a study to observe the effects on the healing of chronic DFUs between F-ESWT and Hyperbaric Oxygen Therapy (HBOT) [14]. Doppler imaging was used to determine the acute perfusion levels of each wound. The study reported a significant increase in perfusion for the F-ESWT cohort $(\mathrm{N}=44)$ from an average of 0.56 to $0.74(\mathrm{p}<0.001)$. The perfusion rates in the HBOT cohort $(\mathrm{N}=40)$ actually decreased over the course of the 8-week trial from an average of 0.59 to $0.50(p=0.916)$.

In the present case series, NIRS assessment provided objective evidence to suggest that F-ESWT administered to the wound bed improves microcirculation. All fifteen wounds demonstrated significantly increased tissue oxygen saturation levels within the central wound bed after four consecutive weekly doses of F-ESWT. The NIRS device used in this case series, Snapshot ${ }_{N I R}$, calculates $\mathrm{S}_{\mathrm{t}} \mathrm{O}_{2}$ by measuring the absorption of red and near-infrared wavelengths. Using a proprietary algorithm, it delineates the separate and distinct spectral signatures in the visible and near infrared light spectrums of oxygenated hemoglobin and deoxygenated hemoglobin to determine the proportion of each in the sampled tissue [22, 23]. A study by Serena et al compared tissue oxygen saturation measurements of NIRS and transcutaneous oxygen measurement TCOM, which is the current gold standard [24]. Results showed a strong correlation between measurements from each device.

While correlation of increased wound bed $\mathrm{S}_{\mathrm{t}} \mathrm{O}_{2} \%$ and reduction in wound size was not seen in this case series, a statistically significant difference in reduction in wound area with use of F-ESWT compared to standard of care was also not noted in a systematic review and meta-analysis of ESWT in the treatment of diabetic foot ulceration by Huang et al. [25]. Nonetheless, those authors did note shorter healing times for the DFUs treated with F-ESWT. In Sheenan et al.'s landmark study [26], a mean absolute reduction in wound size of $1.5 \mathrm{~cm}^{2}$ at four weeks was noted in those patients that healed. These results demonstrated that percent change in wound area of a diabetic foot ulceration over a 4-week period of treatment was a robust predictor of complete healing at 12-weeks. In the present case series, 14 of the 15 chronic wounds (mean duration 12.3 weeks) demonstrated a greater than $1.5 \mathrm{~cm}^{2}$ reduction of wound area over the four-week study period, indicating that they were now on a healing trajectory. Thus, the increase in central wound bed tissue oxygenation seen in this study and Huang's, may help explain the reason for shorter times to healing despite a non-statistically significant correlation with reduction in wound size.

\section{Limitations}

Major limitations of this case series are that it is a single-center study with a small sample size, short treatment duration, and no control group. In our study sample, increases in wound bed $\mathrm{S}_{\mathrm{t}} \mathrm{O}_{2} \%$ did not correlate significantly with a finite reduction in overall wound size. However, the results of the t-tests and signed rank tests performed for change in $\mathrm{S}_{\mathrm{t}} \mathrm{O}_{2} \%$ and change in wound area resulted in statistical significance with a 95\% CI that was far from zero. These results suggest that the increase in $\mathrm{S}_{\mathrm{t}} \mathrm{O}_{2} \%$ and reduction in wound area seen from baseline to follow up was not due to chance and pursuing further investigation into a possible relationship between $\mathrm{S}_{\mathrm{t}} \mathrm{O}_{2} \%$ and wound size reduction in conjunction with F-ESWT therapy may be worthwhile. Additionally, the treatment period was limited to five weeks (four consecutive weekly treatments) which may not be sufficient for determination of the full benefit of F-ESWT treatment. Typical wound care trials that examine for healing efficacy of a wound care therapy assess wounds over a period of 12 weeks. While a four-week treatment schedule in this study was based on Sheehan's finding that percent change in wound area at 4 weeks is a robust predictor of a wound that is on a healing trajectory [26], future studies with a longer duration of treatment may prove valuable. Lastly, as a pilot case series the authors did not include a control group. However, a control group would provide valuable information to aid in determining if F-ESWT therapy is the cause of improved microcirculation, and possibly increased wound size reduction beyond that obtained with standard of care treatment alone.

\section{Conclusions}

The results of this case series suggest that F-ESWT therapy supports neovascularization resulting in increased oxygen saturation level in wounded tissues, potentially facilitating a healing trajectory in chronic wounds of the lower extremity in patients with diabetes. The NIRS device proved to be a very user-friendly point of care imaging device to objectively track weekly wound tissue oxygenation saturation progress. Use of F-ESWT in conjunction with NIRS imaging, may prove a beneficial additional treatment modality for chronic lower extremity ulcerations and other wound types.

\section{Author Contributions}

Conceptualization, W.C..; methodology, W.C.; validation, S.C.; formal analysis, G.M.; investigation, W.C.; data curation, S.C.; writing - original draft preparation, W.C., S.C., G.M., V.M.; writing - review and editing, S.C., V.M.; visualization, S.C.; supervision, W.C..; project administration, W.C., S.C.; funding acquisition, W.C.; all authors-W.C., S.C., G.M., V.M. - read and approved the final version of the manuscript to be published. 


\section{Data Availability Statement}

Data are available upon reasonable request.

\section{Conflicts of Interest}

The authors declare that they have no competing interests.

\section{Acknowledgements}

The authors are grateful to Pete Stegagno and Liz Newell for their technical support. Support was provided by SANUWAVE through the donation of the dermaPACE ${ }^{\circledR}$ System, and by Kent Imaging in the form of the Snapshot NIR $_{\text {device. Publication support was provided }}$ by Kent Imaging.

\section{References}

[1] Centers for Disease Control and Prevention. National Diabetes Statistics Report, 2020. Atlanta, GA: Centers for Disease Control and Prevention, US Department of Health and Human Services; 2020 .

[2] Vuorisalo S, Venermo M, Lepäntalo M. Treatment of diabetic foot ulcers. J Cardiovasc Surg (Torino). 2009; 50 (3): 275-291.

[3] Mavrogenis AF, Megaloikonomos PD, Antoniadou T, et al. Current concepts for the evaluation and management of diabetic foot ulcers. EFORT Open Rev. 2018; 3 (9): 513-525. Published 2018 Sep 27. doi: 10.1302/2058-5241.3.180010.

[4] Barnes JA, Eid MA, Creager MA, Goodney PP. Epidemiology and Risk of Amputation in Patients with Diabetes Mellitus and Peripheral Artery Disease. Arterioscler Thromb Vasc Biol. 2020; 40 (8): 1808-1817. doi: 10.1161/ATVBAHA.120.314595.

[5] Kuo YR, Wu WS, Hsieh YL, et al. Extracorporeal shock wave enhanced extended skin flap tissue survival via increase of topical blood perfusion and associated with suppression of tissue pro-inflammation. J Surg Res. 2007; 143 (2): 385-392. doi: $10.1016 /$ j.jss.2006.12.552.

[6] Cole W, Coe S. Using a non-invasive pulsed acoustic cellular expression system to promote angiogenesis in chronic wounds. Today's Wound Clinic. Oct; 13 (10): 16-20.

[7] Wang CJ. An overview of shock wave therapy in musculoskeletal disorders. Chang Gung Med J. 2003; 26 (4): 220-232.

[8] Rompe JD, Hopf C, Küllmer K, Heine J, Bürger R, Nafe B. Low-energy extracorporal shock wave therapy for persistent tennis elbow. Int Orthop. 1996; 20 (1): 23-27. doi: $10.1007 / \mathrm{s} 002640050021$.

[9] Neidrauer M, Zubkov L, Weingarten MS, Pourrezaei K, Papazoglou ES. Near infrared wound monitor helps clinical assessment of diabetic foot ulcers. J Diabetes Sci Technol. 2010; 4 (4): 792-798. Published 2010 Jul 1. doi: $10.1177 / 193229681000400404$

[10] Khaodhiar L, Dinh T, Schomacker KT, et al. The use of medical hyperspectral technology to evaluate microcirculatory changes in diabetic foot ulcers and to predict clinical outcomes. Diabetes Care. 2007; 30 (4): 903-910. doi: 10.2337/dc06-2209.
[11] Nouvong A, Hoogwerf B, Mohler E, Davis B, Tajaddini A, Medenilla E. Evaluation of diabetic foot ulcer healing with hyperspectral imaging of oxyhemoglobin and deoxyhemoglobin. Diabetes Care. 2009; 32 (11): 2056-2061. doi: $10.2337 / \mathrm{dc} 08-2246$.

[12] Tandara AA, Mustoe TA. Oxygen in wound healing--more than a nutrient. World J Surg. 2004; 28 (3): 294-300. doi: 10.1007/s00268-003-7400-2.

[13] Gallagher KA, Liu ZJ, Xiao M, et al. Diabetic impairments in NO-mediated endothelial progenitor cell mobilization and homing are reversed by hyperoxia and SDF-1 alpha. J Clin Invest. 2007; 117 (5): 1249-1259. doi: 10.1172/JCI29710.

[14] Wang CJ, Wu RW, Yang YJ. Treatment of diabetic foot ulcers: a comparative study of extracorporeal shockwave therapy and hyperbaric oxygen therapy. Diabetes Res Clin Pract. 2011; 92 (2): 187-193. doi: 10.1016/j.diabres.2011.01.019.

[15] Cooper B, Bachoo P. Extracorporeal shock wave therapy for the healing and management of venous leg ulcers. Cochrane Database Syst Rev. 2018; 6 (6): CD011842. Published 2018 Jun 11. doi: 10.1002/14651858.CD011842.pub2.

[16] Stojadinovic A, Elster EA, Anam K, et al. Angiogenic response to extracorporeal shock wave treatment in murine skin isografts. Angiogenesis. 2008; $11 \quad$ (4): 369-380. doi: 10.1007/s10456-008-9120-6.

[17] Wang CJ, Kuo YR, Wu RW, et al. Extracorporeal shockwave treatment for chronic diabetic foot ulcers. J Surg Res. 2009; 152 (1): 96-103. doi: 10.1016/j.jss.2008.01.026.

[18] Krokowicz L, Klimczak A, Cwykiel J, Mielniczuk M, Grykien C, Siemionow M. Pulsed acoustic cellular expression as a protective therapy against $\mathrm{I} / \mathrm{R}$ injury in a cremaster muscle flap model. Microvasc Res. 2012; 83 (2): 213-222. doi: 10.1016/j.mvr.2011.11.005.

[19] Krokowicz L, Cwykiel J, Klimczak A, Mielniczuk M, Siemionow M. Pulsed acoustic cellular treatment induces expression of proangiogenic factors and chemokines in muscle flaps. $J$ Trauma. 2010; 69 (6): 1448-1456. doi: 10.1097/TA.0b013e3181ddd067.

[20] Rudic RD, Shesely EG, Maeda N, Smithies O, Segal SS, Sessa WC. Direct evidence for the importance of endothelium-derived nitric oxide in vascular remodeling. $J$ Clin Invest. 1998; 101 (4): 731-736. doi: 10.1172/JCI1699.

[21] De Sanctis MT, Belcaro G, Nicolaides AN, et al. Effects of shock waves on the microcirculation in critical limb ischemia (CLI) (8-week study). Angiology. 2000; 51 (8 Pt 2): S69-S78. doi: $10.1177 / 000331970005100809$.

[22] Boezeman RP, Becx BP, van den Heuvel DA, Ünlü C, Vos JA, de Vries JP. Monitoring of Foot Oxygenation with Near-infrared Spectroscopy in Patients with Critical Limb Ischemia Undergoing Percutaneous Transluminal Angioplasty: A Pilot Study. Eur J Vasc Endovasc Surg. 2016; 52 (5): 650-656. doi: 10.1016/j.ejvs.2016.07.020.

[23] Stranc MF, Sowa MG, Abdulrauf B, Mantsch HH. Assessment of tissue viability using near-infrared spectroscopy. Br J Plast Surg. 1998; 51 (3): 210-217. doi: 10.1054/bjps.1997.0088.

[24] Serena TE, Yaakov R, Serena L, Mayhugh T, Harrell K. Comparing near infrared spectroscopy and transcutaneous oxygen measurement in hard-to-heal wounds: a pilot study. $J$ Wound Care. 2020; 29 (Sup6): S4-S9. doi: 10.12968/jowc.2020.29.Sup6.S4. 
[25] Huang Q, Yan P, Xiong H, et al. Extracorporeal Shock Wave Therapy for Treating Foot Ulcers in Adults With Type 1 and Type 2 Diabetes: A Systematic Review and Meta-Analysis of Randomized Controlled Trials. Can J Diabetes. 2020; 44 (2): 196-204.e3. doi: 10.1016/j.jcjd.2019.05.006.
[26] Sheehan P, Jones P, Caselli A, Giurini JM, Veves A. Percent change in wound area of diabetic foot ulcers over a 4-week period is a robust predictor of complete healing in a 12-week prospective trial. Diabetes Care. 2003; 26 (6): 1879-1882. doi: 10.2337/diacare.26.6.1879. 